\title{
Distributed observations of wind direction using microstructures attached to actively heated fiber-optic cables
}

\author{
Karl Lapo $^{1,2}$, Anita Freundorfer ${ }^{1}$, Lena Pfister ${ }^{1}$, Johann Schneider ${ }^{1}$, John Selker ${ }^{3}$, and Christoph Thomas ${ }^{1,2}$ \\ ${ }^{1}$ Department of Micrometeorology, University of Bayreuth, Bayreuth, Germany \\ ${ }^{2}$ Bayreuth Center of Ecology and Environmental Research, Bayreuth, Germany \\ ${ }^{3}$ Department of Biological and Ecological Engineering, Oregon State University, Corvallis, Oregon, USA
}

Correspondence: Karl Lapo (karl.lapo@uni-bayreuth.de)

Received: 8 May 2019 - Discussion started: 25 November 2019

Revised: 11 February 2020 - Accepted: 23 February 2020 - Published: 1 April 2020

\begin{abstract}
The weak-wind boundary layer is characterized by turbulent and submesoscale motions that break the assumptions necessary for using traditional eddy covariance observations such as horizontal homogeneity and stationarity, motivating the need for an observational system that allows spatially resolving measurements of atmospheric flows near the surface. Fiber-optic distributed sensing (FODS) potentially opens the door to observing a wide range of atmospheric processes on a spatially distributed basis and to date has been used to resolve the turbulent fields of air temperature and wind speed on scales of seconds and decimeters. Here we report on progress developing a FODS technique for observing spatially distributed wind direction. We affixed microstructures shaped as cones to actively heated fiber-optic cables with opposing orientations to impose directionally sensitive convective heat fluxes from the fiber-optic cable to the air, leading to a difference in sensed temperature that depends on the wind direction. We demonstrate the behavior of a range of microstructure parameters including aspect ratio, spacing, and size and develop a simple deterministic model to explain the temperature differences as a function of wind speed. The mechanism behind the directionally sensitive heat loss is explored using computational fluid dynamics simulations and infrared images of the cone-fiber system. While the results presented here are only relevant for observing wind direction along one dimension, it is an important step towards the ultimate goal of a full three-dimensional, distributed flow sensor.
\end{abstract}

\section{Introduction}

Laser pulses sent along a fiber-optic cable scatter back along the path of the fiber with a temperature-dependent shift in frequency, providing a powerful geophysical sensing technique called distributed temperature sensing (DTS) (Selker et al., 2006; Tyler et al., 2009). The principle behind DTS has been used to observe a wide range of geophysical processes, and aerial deployments of DTS are a promising avenue for observing atmospheric processes on a distributed basis (Pfister et al., 2016; Thomas et al., 2012; Zeeman et al., 2015). Previous work with atmospheric DTS has demonstrated the ability to observe atmospheric temperatures (Thomas et al., 2012), wet bulb temperature (Euser et al., 2014; Schilperoort et al., 2018), solar radiation (Sigmund et al., 2017; Petrides et al., 2011), and wind speed (Sayde et al., 2015) at a fine spatial and temporal resolution. We refer to this broader application of DTS technology as fiber-optic distributed sensing (FODS).

FODS has the potential to fill the missing scales between point observations and remote sensing. FODS can provide observations of atmospheric variables at temporal resolution as fine as $1 \mathrm{~s}$ and spatially distributed observations on scales from tens of centimeters to kilometers. In particular, FODS is ideally suited for observing turbulence, especially during weak-wind conditions. Weak-wind boundary layers break many of the assumptions that underlie eddy covariance techniques (Thomas, 2011; Cheng et al., 2017; Pfister et al., 2019), which forms an obstacle for understanding the dynamics of turbulence during these conditions. For instance, eddy covariance relies on the ergodic hypothesis, 
the assumption that time and space averages converge under horizontally homogeneous and stationary conditions (Taylor, 1938). From this assumption, the time-averaged flow can be used to infer the spatially averaged fluxes and turbulent properties. However, weak-wind boundary layers break this critical assumption, thereby limiting the inferences we can make about the nature of turbulence from point observations alone, even within exceptionally dense observation networks (Pfister et al., 2019; Mahrt et al., 2009). Further, weak-wind boundary layers violate the assumptions behind similarity theory, with nonlocal and intermittent fluxes (Sun et al., 2012, 2015), creating substantial problems for climate, weather, and land models which rely on similarity theory to simulate turbulent fluxes at the land surface (Holtslag et al., 2013).

The ability to observe spatially distributed wind direction, in addition to wind speed and temperature, at a fine spatial and temporal scale near the surface, would be a powerful technique for studying atmospheric turbulence. However, prior work has only been able to observe the magnitude of wind speed normal to the fiber, not the direction (Pfister et al., 2019; Sayde et al., 2015; Ramshorst et al., 2019). The approach for observing flow direction with FODS explored in this study is based upon the hypothesis that microstructures with opposite orientations placed on paired, actively heated fiber-optic cables impart a directionally sensitive convective heat loss (Sect. 2.1).

Here, we present results from a series of wind tunnel experiments that demonstrate the basic feasibility of observing wind direction with FODS. Additionally, an empirical expression for describing the FODS signal of wind direction was developed (Sect. 2.1), parameters that govern the magnitude of the signal were tested (Sect. 3.2), the uncertainty and scale of the wind direction signal were evaluated (Sect. 3.3), and the mechanism behind the wind direction signal was verified (Sect. 3.4). Finally, these results are discussed within the context of the remaining challenges for observing spatially distributed wind direction in an environmental application (Sect. 3.5).

\section{Methods}

\subsection{Motivating the microstructure approach}

Raman spectra DTS operates on the principle of temperaturedependent backscattering of photons at a higher and lower frequency than the original laser pulse. The reader is referred to Selker et al. (2006) and Tyler et al. (2009) for a detailed description of the operating principal. This temperature dependency can be used to observe air temperature directly. Wind magnitude orthogonal to the cable can be observed using the temperature difference between an active, resistively heated cable and a paired, unheated cable, similar to the principle of hot-wire anemometery (Sayde et al., 2015; Ramshorst et al.,
2019). Actively heating the cable causes it to be warmer than the atmosphere; thus the convective heat flux cools the heated cable: stronger winds cause a larger, cooling convective heat flux and a smaller temperature difference between the paired cables (Sayde et al., 2015).

To observe wind direction, we propose a similar approach combining the active heating with microstructures printed directly on the fiber-optic cable (FOC). The underlying assumption is that applying asymmetric microstructures with opposite orientations to paired, actively heated cables induces directional differences in the turbulent flow around the microstructures and thus in the convective heat loss from the FOC to the air. This difference in convective heat loss results in a temperature difference between the two cables that can be sensed by FODS (see Fig. 1).

The convective heat loss from a surface with roughness elements can be written as (Owen and Thomson, 1962)

$Q_{h}=\rho u_{*} c_{\mathrm{p}}\left(T_{\mathrm{a}}-T_{\mathrm{s}}\right) \alpha^{-1} \operatorname{Re}_{*}^{-m} \operatorname{Pr}^{-n}$,

where $\rho$ is the density of air, $c_{\mathrm{p}}$ is the specific heat of dry air, $T_{\mathrm{a}}$ is the temperature of the air, $T_{\mathrm{s}}$ is the temperature of the fiber, $\alpha$ is an empirical constant related to the roughness of the surface, and $P r$ is the Prandtl number. $m$ and $n$ are empirical constants. $R e_{*}$ is the roughness Reynolds length defined as

$R e_{*}=\frac{u_{*} h}{v}$,

where $u *$ is the friction velocity, $h$ is the "sand-equivalent height" of the roughness elements or the thickness of the surface layer (Owen and Thomson, 1962), and $v$ is the kinematic viscosity. Combining Eqs. (1) and (2) yields

$Q_{h}=\rho u_{*}^{1-m} c_{\mathrm{p}}\left(T_{\mathrm{a}}-T_{\mathrm{s}}\right) \alpha^{-1}\left(\frac{h}{v}\right)^{-m} \operatorname{Pr}^{-n}$.

Cones pointing into the flow should have a lower equivalent roughness height than cones pointing with the flow as a result of changing from a streamlined shape into a bluff shape. Consequently, actively heated cables with microstructures of opposite orientations should have different cooling convective heat fluxes and resulting temperatures.

The difference in the convective heat flux along each cable is manifested as a temperature difference between the cables from which we define the wind direction signal:

$\Delta T=T_{\text {right }}-T_{\text {left }}$.

Cables with microstructures pointing into the flow (Fig. 1) should have a smaller (cooling) convective heat flux and higher temperature $\left(T_{\text {right }}\right)$ compared to cables with microstructures pointing with the flow, which should have a larger convective heat flux and resulting lower temperature $\left(T_{\text {left }}\right)$. In the displayed case (Fig. 1) $\Delta T>0$ would indicate a wind direction from the right, while $\Delta T<0$ indicates a wind direction from the left. 


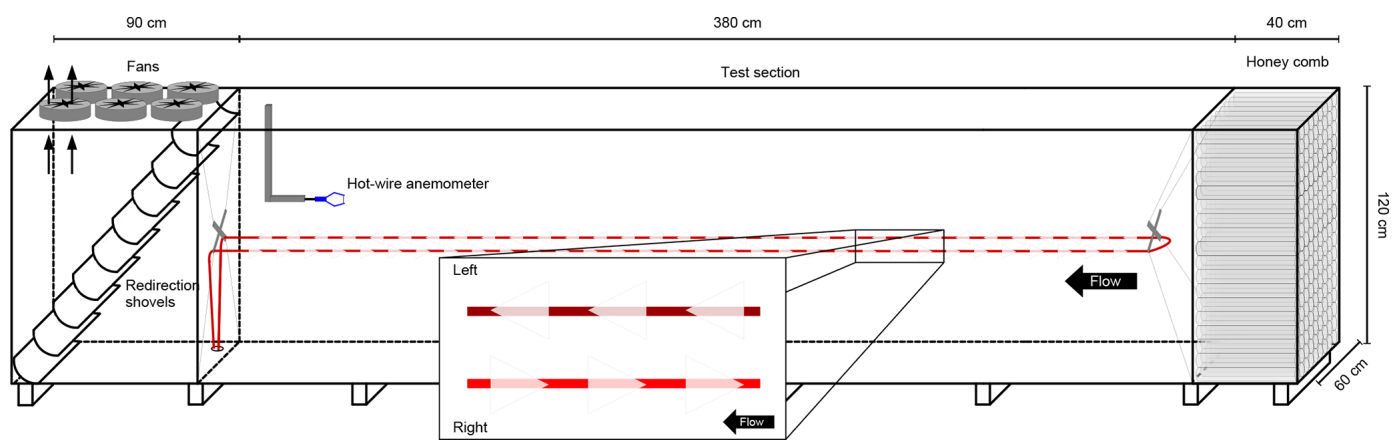

Figure 1. Schematic of the low-speed wind tunnel used to test the microstructure approach to detecting wind direction. The subset highlights the cone orientation relative to the mean flow of the tunnel. The FOC with left-pointing cones is cooler than the FOC with the right-pointing cones.

To derive a functional form for $\Delta T$ as a function of wind speed, we substitute $T_{\text {right }}$ and $T_{\text {left }}$ into Eq. (1) and subtract the two quantities.

$$
\begin{aligned}
Q_{\text {right }}-Q_{\text {left }} & =\rho c_{\mathrm{p}}\left(\frac{h}{v}\right)^{-m} u_{*}^{(1-m)} \\
& \left(\frac{\left(T_{\mathrm{a}}-T_{\text {right }}\right)}{\alpha_{\text {right }}}-\frac{\left(T_{\mathrm{a}}-T_{\text {left }}\right)}{\alpha_{\text {left }}}\right)
\end{aligned}
$$

Solving the equation uniquely for $T_{\text {left }}-T_{\text {right }}$ requires knowledge of the form of the friction terms, $\alpha_{\text {left }}$ and $\alpha_{\text {right }}$. Without an exact expression for these terms Eq. (5) reduces to

$$
\begin{aligned}
\left(\frac{T_{\text {left }}}{\alpha_{\text {left }}}-\frac{T_{\text {right }}}{\alpha_{\text {right }}}\right) & =\frac{Q_{\text {right }}-Q_{\text {left }}}{\rho c_{\mathrm{p}}\left(\frac{h}{\nu}\right)^{-m}} u_{*}^{m-1} \\
& -T_{\mathrm{a}}\left(\frac{1}{\alpha_{\text {right }}}-\frac{1}{\alpha_{\text {left }}}\right) .
\end{aligned}
$$

Equation (6) suggests that $\Delta T$ is nonlinearly related to wind speed, $U$, since $u_{*}$ and $U$ are linearly related quantities (Stull, 1988). Equation (6) also implies that $U$ and $\Delta T$ have a power law relationship with a negative exponent, as $m$ should be less than 0.5. While expressions for the friction terms are unknown, some sort of nonlinear, decreasing relationship between $\Delta T$ and $U$ is anticipated. Accordingly, we also test an exponential decay model as a competing representation of the nonlinear relationship.

\subsection{Instruments and wind tunnel}

The microstructure approach to distributed observations of wind direction was tested in a controlled wind tunnel environment. The wind tunnel was designed to provide small turbulence intensity for low velocity flows. The suck-through wind tunnel test section was $3.0 \mathrm{~m}$ long, $0.6 \mathrm{~m}$ wide, and $1.2 \mathrm{~m}$ tall. At the entrance of the wind tunnel the flow was straightened and external turbulence was combed out using a honeycomb section made from $0.20 \mathrm{~m}$ long pipes with a $0.05 \mathrm{~m}$ radius. The flow was allowed to settle over $0.6 \mathrm{~m}$ before entering the test section. The tunnel was lined with $0.03 \mathrm{~m}$ thick insulating polyurethane boards to eliminate spatial differences in surface temperature and thus provided a coherent radiative environment within the tunnel, which minimizes differences in the longwave radiative transfer that arises from even subtle differences in surface temperature and emissivity. Deviations of longwave radiation parallel to the cable can cause artifacts in the DTS.

A sonic anemometer (Model CSAT3, Campbell Scientific Inc., Logan, UT, USA) was used to pre-characterize the flow by moving the instrument throughout the tunnel both in transects along the tunnel and in selected planes orthogonal to the along-tunnel dimension. The tunnel was controlled to yield consistent wind speeds for each test with along-flow velocities as given in Table 1. Each parameter was tested with $10 \mathrm{~min}$ of observations using a flow that did not vary in time. The center of the wind tunnel was free from the influence of a shallow wall boundary layer. At the back of the tunnel there was some enhancement of turbulent mixing due to deflection towards the fans, which was excluded from further analysis. Horizontal turbulent intensity, the ratio of the standard deviation of the horizontal wind components to the mean velocity, varied between 0.005 and 0.01 for the lowest to highest wind tunnel velocities. The friction velocity along the central axis of the wind tunnel ranged from $0.0025 \mathrm{~m} \mathrm{~s}^{-1}$ for the lowest wind speeds to $0.02 \mathrm{~m} \mathrm{~s}^{-1}$ for the highest wind speeds. The turbulence within the tunnel was substantially lower compared to atmospheric flows (e.g., Friedrich et al., 2012) and may impact direct transfer of wind tunnel results to field conditions.

The time-averaged wind speed during the 10 min tests with the FOC was monitored using a one-dimensional hot-wire anemometer (Model, TA300, Trotec, Heinsberg, Germany) with a precision of $\pm 0.2 \mathrm{~m} \mathrm{~s}^{-1}$. To provide an independent measurement of the fiber temperatures, a high-resolution thermal infrared camera was used to record its brightness temperature (Model PI640, Optris, Berlin, Germany). The camera observes the wavelengths of 7.5 to $13 \mu \mathrm{m}$ with a pixel resolution of $640 \times 480$ and an accuracy of $\pm 2{ }^{\circ} \mathrm{C}$. The cam- 
Table 1. Tested parameters values.

\begin{tabular}{ll}
\hline Variable & Values \\
\hline Cone size & $0.012,0.016 \mathrm{~m}$ \\
Cone spacing & $0.02,0.05,0.1 \mathrm{~m}$ \\
Cone aspect ratio & $1: 1$ (regular), $1: 2$ (long and thin), \\
& $2: 1$ (short and wide) \\
Heating rate & $0.5,1.5,2.5 \mathrm{~W} \mathrm{~m}^{-1}$ \\
Mean wind speed & $0.35,0.91 .8,3.8 \mathrm{~m} \mathrm{~s}^{-1}$ \\
\hline
\end{tabular}

era was placed on the floor of the wind tunnel looking up at the FOCs such that the pixel resolution was $0.00025 \mathrm{~m}$. Pictures of the fiber and microstructure brightness temperatures were acquired at $1 \mathrm{~Hz}$ and averaged over $10 \mathrm{~s}$. In order to avoid angular effects of the emitted thermal radiation, the cones and fiber were coated with infrared paint (Washable thermographic paint for special applications, LabIR, Pilsen, Czech Republic) that has a known emissivity of 0.94 to 0.97 for viewing angles 60 to $5^{\circ}$, respectively.

\subsection{Fiber-optic array}

One continuous FOC was deployed in the test section, parallel to the flow within the wind tunnel (Fig. 1). The cable was mounted within the tunnel using square aluminum crosses with a width of $7.5 \mathrm{~cm}$ and was gently looped around the back of the crosses to avoid sharp bends which cause a signal loss (Selker et al., 2006). The FOC had a $0.82 \mathrm{~mm}$ stainless steel sheath with a $0.15 \mathrm{~mm}$ PVC coating, yielding a total outer diameter of $1.12 \mathrm{~mm}$ and the actual fiber-optic cable was loosely buffered and gel-filled (Model C-Tube, Brugg, Brugg, Switzerland). The cable was heated electrically by applying a current to the high-resistance $\left(2.3 \Omega \mathrm{m}^{-1}\right)$ stainless steel sheath. Microstructures were mounted in opposing directions on the looped cable (zoomed in region Fig. 1).

Fiber temperature was observed using a high-resolution DTS instrument (Model $5 \mathrm{~km}$ Ultima, Silixa, London, UK). This DTS device is capable of fine-scale distributed temperature observations with a sampling resolution of $0.127 \mathrm{~m}$ with a temporal resolution of $\approx 1 \mathrm{~s}$. The implications of instrument noise on the wind direction approach is discussed in Sect. 3.3.

The intensity of the back-scattered light was converted to a temperature using calibrated parameters that vary with instrument temperature and fiber properties. We explicitly calculate these parameters through a matrix inversion of the back-scatter equation using three reference sections with a single-ended approach (Hausner et al., 2011). This calibration technique eliminates effects from differential attenuation and instrument properties that can vary with time.

The reference sections are composed of warm and cold calibration baths. The cables were deployed in the calibration baths both prior to entering the wind tunnel and after the fiber exits the tunnel, yielding two temperatures at four lo- cations along the fiber. Three of these calibration sections were used to solve for the calibrated parameters with the fourth withheld for characterizing the instrument uncertainty. Each reference bath was well-mixed using aquarium pumps to avoid stratification. The fiber was loosely coiled within the baths such that they did not contact the bath walls. Two class-A PT100s, with an accuracy of $0.15^{\circ} \mathrm{C}$, were deployed in each calibration bath. After calibration, the DTS had a root mean square error of $0.61^{\circ} \mathrm{C}(n=4300000)$ when evaluated against the temperature of the reference bath, in line with the published accuracy from the manufacturer. We used this error as an estimate of the instrument uncertainty (Sect. 3.3).

\subsubsection{Heating}

The heating of the FOC was provided by a high-precision heating unit (Model Heat Pulse System, Silixa, London, United Kingdom) which applies a known heating rate per section of cable. Multiple heating rates were tested (Table 1) as previous work has suggested that heating rate can influence the accuracy of FODS of wind variables (Sayde et al., 2015) as the convective heat loss is a linear function of the temperature difference between the cable and air temperatures.

\subsubsection{Microstructures}

We used 3-D-printed (Model Form 2, Formlabs, Berlin, Germany) cones that can be affixed to the FOC. Cone base width was selected as 12 and $16 \mathrm{~mm}$, with the ratio of width : height varying from $1: 1$ (as tall as it is long), $1: 2$ (long and thin), to $2: 1$ (short and wide; see Table 1). These cones were then affixed to the paired, heated cables with each cable having cones oriented in the opposite direction from the other, as shown in Fig. 1. The distance between cones was varied between 2 and $10 \mathrm{~cm}$ (Table 1). While we solely used cones for this study, we speculate that additional shapes may be used to achieve a similar directional dependence.

\subsection{Numerical simulations}

Computational fluid dynamics (CFD) simulations were completed to inform the initial design decisions of the microstructures and to verify the observed heat transfer mechanism (Sect. 3.4). We used the OpenFOAM computational fluid dynamics software (https://www.openfoam.com, last access: 30 March 2020) with the simpleFoam solver and the standard $k-\epsilon$ turbulence model for doing a 3-D simulation of the flow along a fiber with microstructures. The simulations were done using a long enough piece of fiber such that the flow could adjust to the microstructures. We tested the heating rate, microstructure size, aspect ratio, and spacing each at a variety of wind speeds. The initial CFD simulations allowed the targeting of a specific range of variables. This CFD approach does not simulate heat transfer, so effects like buoyancy are neglected, which is a reasonable assump- 
tion for such small temperature perturbations (Zeitoun et al., 2011).

\section{Results and discussion}

\subsection{Temperature differences}

The results confirmed our initial assumption of directionally sensitive heat loss, and thus cable temperatures, from the cones pointing in different directions (see Fig. 2 for an example for a single test of cone spacing, size, aspect ratio, and heating rate as a function of wind speed). The largest temperature difference between cables coincides with the location of the microstructures (between 0.75 and $2.25 \mathrm{~m}$ ). At the beginning $(x=0 \mathrm{~m})$ and end $(x=3 \mathrm{~m})$ of the test section, the cable temperature exhibits artifacts caused by the support crosses that are used to mount the fiber in the tunnel. The microstructure fibers exhibit a uniform temperature within the test section except at the lowest wind speed, in which a decrease in temperature is observed with length along the tunnel, perhaps as a result of unorganized turbulence within the tunnel at these low wind speeds. The heated fibers cool as the wind speed is increased, as expected.

At all wind speeds in the region with cones, the fiber with cones pointing left (Fig. 1) has a lower temperature than the fiber with cones pointing right. $\Delta T$ is the largest at the lowest wind speeds and becomes small enough at the highest wind speed that the fiber temperatures overlap (shaded regions in Fig. 2d). The effect of the uncertainty in the $\Delta T$ signal is discussed further in Sect. 3.3. The reduction in the $\Delta T$ signal with higher wind speeds may be caused by the enhanced roughness from both microstructure orientations being unable to increase the sensible heat flux beyond some maximum value.

When determining the temperature difference, we examine the test section in which the cone signal is not affected by edge effects ( 0.75 to $2.25 \mathrm{~m}$ along the tunnel). The temperature signal for both fibers is linearly interpolated to a common $x$ coordinate along the tunnel.

\subsection{Optimizing the microstructure configuration}

The temperature signal is defined as the mean difference, in both time and space, according to Eq. (4). A positive $\Delta T$ is expected, as the fiber with left-pointing cones should be warmer than the fiber with the right-pointing cones. The tested parameters were stratified from the most influential (Fig. 3a) to least influential (Fig. 3c) factors. A hyperbolic and an exponential model were fit to the set of best performing parameters (Fig. 3d).

The most influential parameter was the cable heating rate. A heating rate between 1.5 and $2.5 \mathrm{~W} \mathrm{~m}^{-1}$ yields the largest $\Delta T$ with a mean difference of approximately equal to $1.0 \mathrm{~K}$ at the lowest wind speed and $0.3 \mathrm{~K}$ at the highest wind speed (Fig. 3a). Certain combinations of microstructure properties with higher heating rates have a smaller $\Delta T$ than different combinations of microstructure properties with a lower heating rate. However, a larger heating rate leads to a larger $\Delta T$ for a fixed combination of cone size, spacing, and aspect ratio.

The cone spacing was the second most influential factor, largely due to the smallest cone spacing of $0.02 \mathrm{~m}$ (Fig. 3b). The mean $\Delta T$ values for the 0.05 and $0.1 \mathrm{~m}$ spacing were indistinguishable at higher wind speeds, while the $0.02 \mathrm{~m}$ spacing resulted in the highest $\Delta T$. The other parameter values tested, such as cone aspect ratio and size, had a limited effect (Fig. 3c). The exception was the aspect ratio, width : height, of $1: 2$, which led to the smallest $\Delta T$ as the other two aspect ratios converged to the same $\Delta T$ for higher wind speeds. At lower wind speeds, the effect of cone size and aspect had an inconsistent effect. For instance, the $0.016 \mathrm{~m}$ cones had the largest temperature difference for the $1: 1$ cones and the smallest difference for the $2: 1$ cones. This ordering reversed itself at higher wind speeds (Fig. 3c). However, these differences were well within the observational uncertainty for the DTS device and should not be interpreted. From this we conclude that any combination of the 0.012 and $0.016 \mathrm{~m}$ cone sizes and the $1: 1$ and $2: 1$ aspect ratios was appropriate for developing the distributed wind direction observation system.

We further evaluated whether $\Delta T$ is well-described by a power law or exponential decay relationship with wind speed. The power law function outperformed the exponential decay as it has smaller residuals and a lower uncertainty in the fit parameters. Additionally, it was able to describe all sets of tested parameters as a function of wind speed, albeit with different parameter values, while the exponential fit cannot (not shown). The results here suggest that the basic relationship shown in Eq. (6) is applicable. It should be noted that both functions have problematic limiting behavior as the wind speed approaches zero. Further work will be necessary to identify a minimum wind speed threshold at which $\Delta T$ becomes significantly nonzero.

\subsection{Certainty in estimating the wind direction signal}

The wind direction signal, $\Delta T$ is subject to a non-negligible uncertainty from the DTS device. The uncertainty for $\Delta T$ is formulated as

$\delta_{T}=\left(2 \sigma_{\text {DTS }}^{2}\right)^{\frac{1}{2}}$,

where $\delta_{T}$ is the uncertainty in the $\Delta T$ signal and $\sigma_{\mathrm{DTS}}$ is the mean standard error derived from the DTS reference thermometers immersed in the calibration baths of uniform temperature. For the DTS device used in this study, the mean standard error is $0.61^{\circ} \mathrm{C}$ (Sect. 2.3) yielding an uncertainty $\delta_{T}=0.81^{\circ} \mathrm{C}$. From this it follows that any $\Delta T \leq \delta_{T}$ cannot be distinguished from noise.

The distribution of $\Delta T$ in both time and space $(n=$ 20700) for a given experiment was normally distributed 
(a) Mean wind speed $=0.35 \mathrm{~ms}^{-1}$

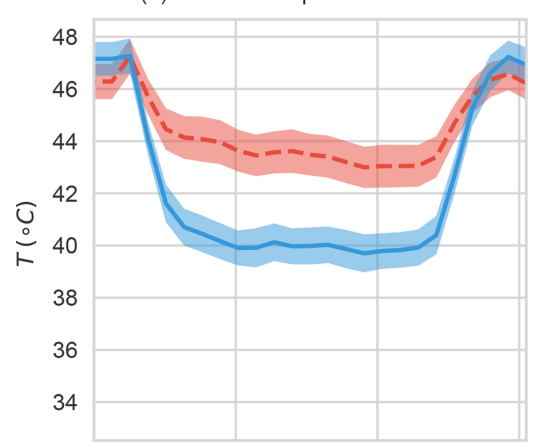

(c) Mean wind speed $=1.8 \mathrm{~m} \mathrm{~s}^{-1}$

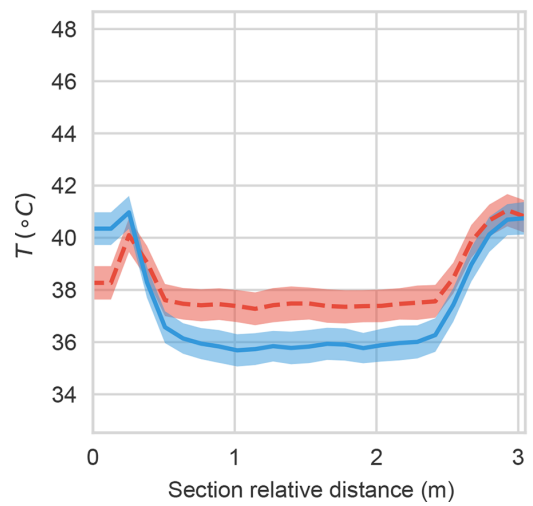

(b) Mean wind speed $=0.9 \mathrm{~ms}^{-1}$

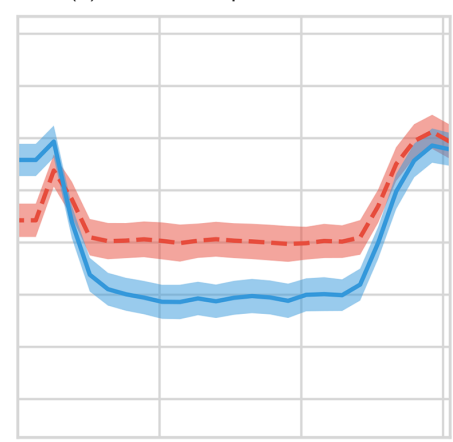

(d) Mean wind speed $=3.8 \mathrm{~ms}^{-1}$

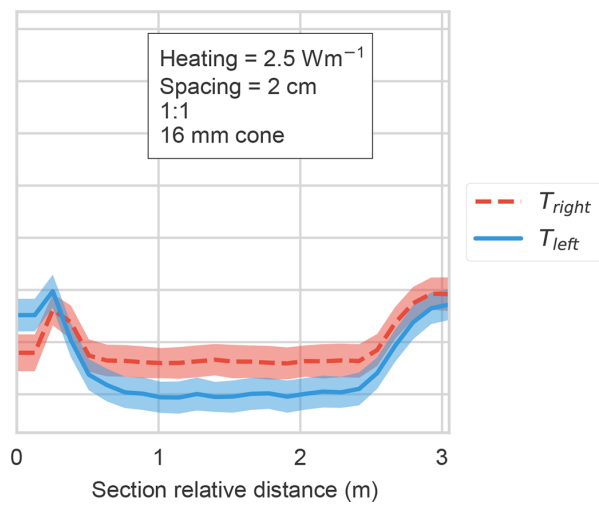

Figure 2. Time-averaged temperature $(10 \mathrm{~min})$ along the test section in the wind tunnel for a horizontal wind speed of (a) 0.35 , (b) 0.9 , (c) 1.8 , and (d) $3.8 \mathrm{~m} \mathrm{~s}^{-1}$ for the parameters listed. The standard deviation of temperature in time for each point along the FOC is shown in the filled colors. The cones are only present between $0.75 \mathrm{~m}$ and 2.25 of the tunnel section. The mean wind direction is in the positive $x$ direction.

around the mean difference (Fig. $4 a, b)$. The strength of the wind direction signal was inversely related to wind speed (Fig. 2) and as a consequence the fraction of $\Delta T \leq \delta$ increased with the wind speed. At the highest wind speed tested, some $\Delta T$ values even changed sign, which would result in an incorrect wind direction estimation (Fig. 4b).

One strategy for improving the accuracy of the wind direction signal is to average the temperature signal from both cables in time and space prior to computing $\Delta T$. A running average of temperature was calculated using a variable number of time (1 s) and/or space $(0.127 \mathrm{~m})$ observations (Fig. $4 \mathrm{c}, \mathrm{d})$. Averaging the temperature signals over longer temporal and spatial intervals than the native resolution of the DTS device reduced the fraction of $\Delta T$ signal below the instrument accuracy and hence increased the fraction of observations suitable for wind direction determination (Fig. 4c). For a given set of microstructure parameters, all wind speeds benefit from averaging the FODS signals. The exception is the lowest wind speed of $0.35 \mathrm{~m} \mathrm{~s}^{-1}$, which effectively always yielded a $\Delta T$ larger than $\delta_{T}$ (Fig. 4c). In this case, the averaging reduces the temporal resolution of the wind detection calculations. All acceptable cone size and aspect ratios found in Sect. 3.2 had similar responses to averaging the FODS signal except the $0.012 \mathrm{~m}$ cones with a $2: 1$ aspect ratio yielded slightly greater uncertainty than other combinations (Fig. 4d).

Any averaging reduces the resolution of the method. Fewer time intervals are necessary for improving the certainty in the $\Delta T$ signal compared to spatial averages for all wind speeds (Fig. 4c). However, an interpretation solely based upon number of averaging intervals may be misleading as the wind direction method is aimed at observing atmospheric turbulence, especially for the weak-wind boundary layer featuring short-lived and small-scale motions. Instead, we seek to find spatial and temporal averages that facilitate the observation of mean direction for a typical eddy length scale during those conditions. Taylor's hypothesis (Taylor, 1938) is commonly employed for estimating the eddy scale that can be resolved with a particular instrument resolution. With Taylor's hypothesis timescales can be converted into spatial scales by assuming a relationship between temporal and spatial gradients through

$\ell=U \tau$,

where $U$ is the mean wind speed, $\ell$ is the spatial scale of a turbulent eddy, and $\tau$ is the timescale. At a wind speed of $4 \mathrm{~m} \mathrm{~s}^{-1}$, a $4 \mathrm{~s}$ average results in observing eddies of length 
(a) Heating Rate

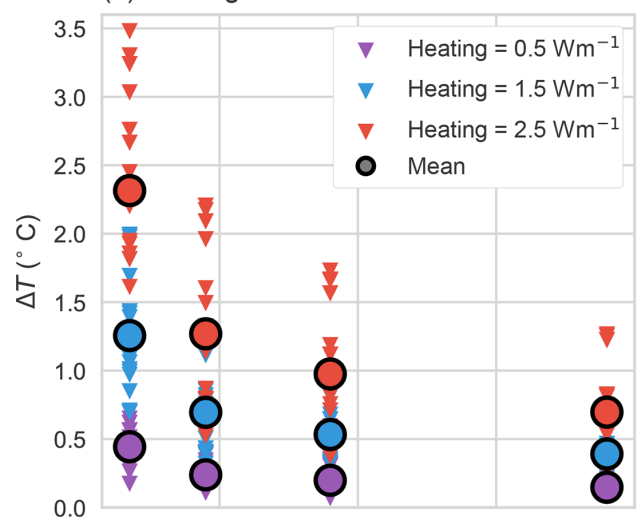

(c) Aspect ratio and cone size

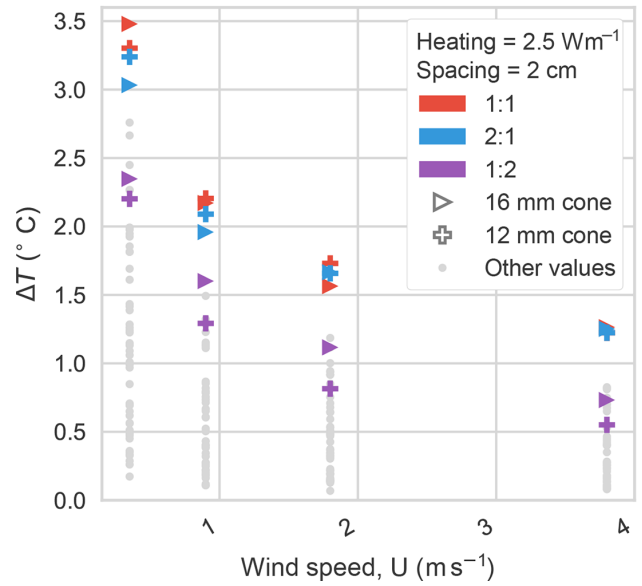

(b) Spacing

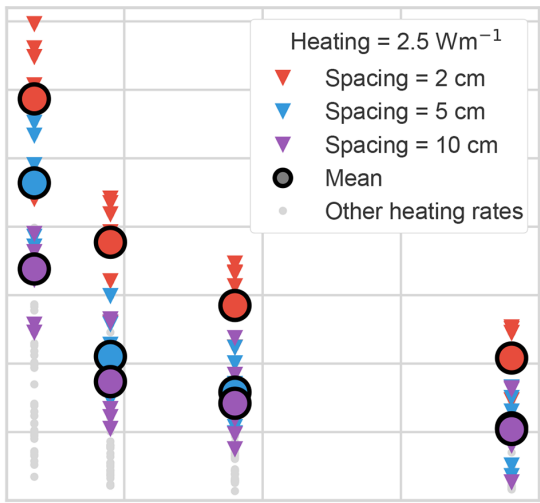

(d) Decay model

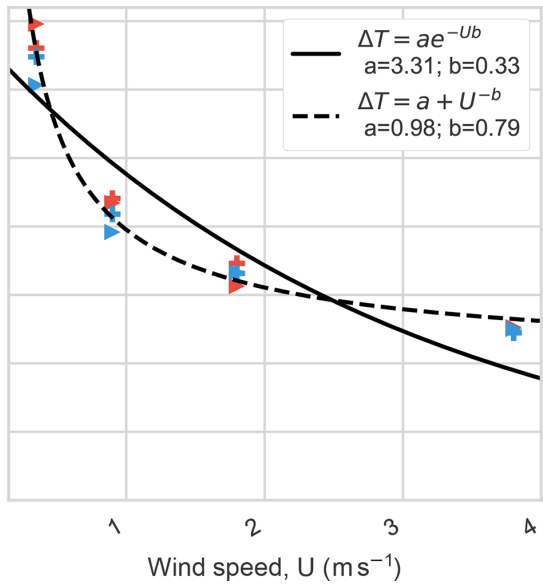

Figure 3. The $\Delta T$ for all combinations of cones and heating rates (see Table 1). The data are stratified using the variable with the largest impact, i.e., temperature difference between coned cable sections pointing in different directions. (a) All temperature differences as a function of wind speed classified by heating rate. (b) The highest heating rate is selected (red points in a) and data are classified according to the spacing between cones. Data with lower heating rates are shown in grey. (c) The $2 \mathrm{~cm}$ spacing and highest heating rates (red points in a and $\mathbf{b}$ ) are selected and then classified according to the cone aspect ratio. The $12 \mathrm{~mm}$ cones are marked with pluses, and the $16 \mathrm{~mm}$ cones are marked with right-pointing triangles. All other heating rates and cone spacings are shown in grey. (d) The best performing parameters are fit with an exponential and power law model (see Sect. 2.1 for details).

scale of $\approx 16 \mathrm{~m}$. This eddy length scale is larger than those commonly found in the weak-wind boundary layer, whereas a spatial average with $n=10$ observations equates to an observation every $1.27 \mathrm{~m}$. Using the DTS temporal resolution, for the same $4 \mathrm{~m} \mathrm{~s}^{-1}$ wind speed, an eddy of length scale $\approx 4 \mathrm{~m}$ can be observed. For this reason, improving the certainty in $\Delta T$ at higher wind speeds through spatial averaging is recommended.

\subsection{Explaining the physical mechanism for directional heat loss}

To provide an independent verification of the FODS signals and investigate the mechanism behind the observational principle, a thermal infrared camera was employed to observe the fiber and cone brightness temperatures (Fig. 5a-c). The use of a paint with a relatively constant emissivity with view- ing angle allows a comparison of brightness temperature between different parts of the microstructure fiber-optic cable setup. The brightness temperature revealed the fine spatial structure of temperatures across the microstructure-cable, from which we inferred differences in the convective heat loss. The brightness temperatures for both the left- and rightpointing cones were cropped from the larger image (Fig. 5a, b) using a threshold value. The differences in temperature between the left- and right-pointing fibers were driven by differences in temperature in two locations. The base of the cones was the coolest part of the cable-cone system with the base of the left-pointing cones being $\approx 2{ }^{\circ} \mathrm{C}$ cooler than the base of the right-pointing cones (Fig. 5c). The cable immediately behind the right-pointing cones, in the lee of the flow, was the warmest point along the fiber and was warmer than the equivalent segment on the left-pointing cones by $>2.5^{\circ} \mathrm{C}$. 
(a) Mean wind speed $=0.35 \mathrm{~ms}^{-1}$

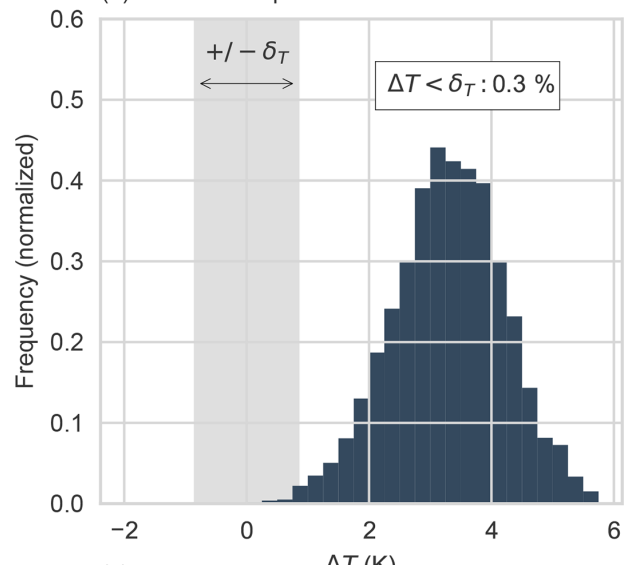

(c)

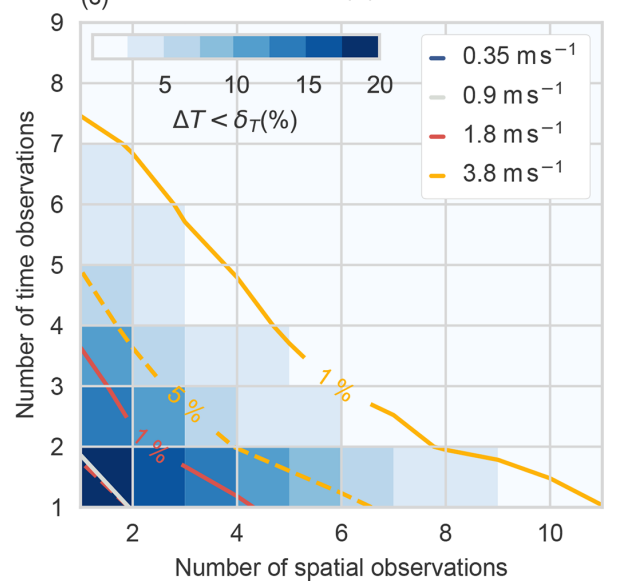

(b) Mean wind speed $=3.8 \mathrm{~ms}^{-1}$

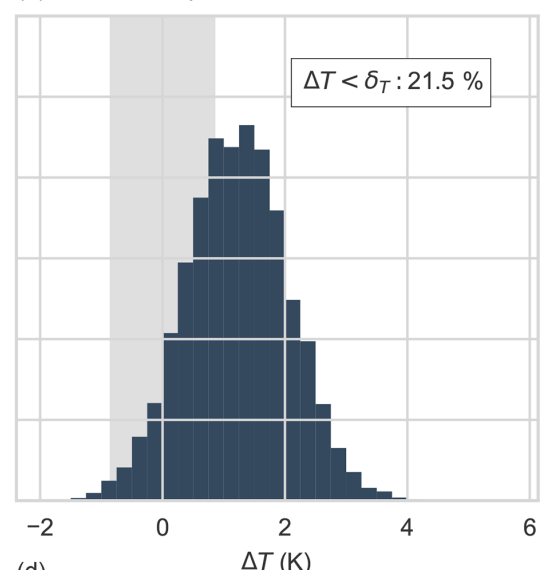

(d)

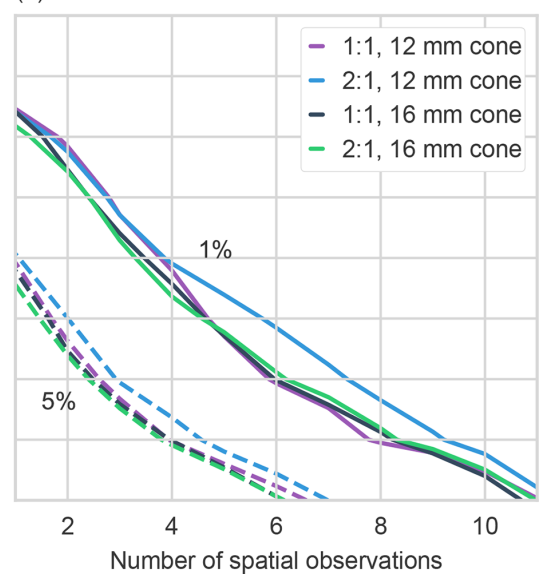

Figure 4. The distribution of temperature differences between cables with opposing cone orientations for the $16 \mathrm{~mm}, 1: 1 \mathrm{cones}$ with $2 \mathrm{~cm}$ spacing and a heating rate of $2.5 \mathrm{~W} \mathrm{~m}^{-1}$ for (a) a wind speed of $0.35 \mathrm{~m} \mathrm{~s}^{-1}$ and (b) a wind speed of $3.8 \mathrm{~m} \mathrm{~s}^{-1}$. The grey region indicates the uncertainty, $\delta_{T}$. The percentage of observations with $\Delta T<\delta_{T}$ is indicated. (c) A 2-D histogram of the percentage of $\Delta T<\delta_{T}$ as a function of averaging interval in time and space for the highest wind speed (same data as in $\mathbf{b}$ ). The contours for the $1 \%$ and $5 \%$ thresholds are indicated for each wind speed. Note that the $0.35 \mathrm{~m} \mathrm{~s}^{-1}$ wind speed is always below the $1 \%$ threshold. (d) The $1 \%$ and $5 \%$ contours for the acceptable cone aspect ratios and sizes at the highest wind speed.

This difference in temperature decreased with length along the cable towards the next pair of cones.

The brightness temperatures suggested that two factors explain the directional sensitivity of the heat loss to the microstructures. First, the low temperatures on the base of the left-pointing cones imply an enhancement of turbulent exchange at the base of the cone relative to the right-pointing cones. Secondly, the high fiber temperatures behind the rightpointing cones imply that the right-pointing cones are sheltering the cable in their lee, reducing the cooling by limiting the convective heat flux. The sheltering effect may partially explain why our findings for the cone spacings of 0.05 and $0.1 \mathrm{~m}$ were indistinguishable (Fig. $3 \mathrm{~b}$ ), as the sheltering occurs over a distance of approximately $0.01-0.02 \mathrm{~m}$. As the spacing increases past the range in which the sheltering occurs, the fibers with right- and left-pointing cones converge to the same temperature (not shown).
The two turbulence features suggested in the brightness temperatures are further demonstrated using CFD simulations for the same fiber setup shown in Fig. 5a and b. The simulated turbulence kinetic energy (TKE) is used as a proxy for the convective heat exchange. TKE is defined as

$\mathrm{TKE}=\frac{1}{2}\left(\overline{\left(u^{\prime}\right)^{2}}+\overline{\left(v^{\prime}\right)^{2}}+\overline{\left(w^{\prime}\right)^{2}}\right)$,

where $u, v$, and $w$ are the three orthogonal wind velocity components and the ' denotes the temporal perturbation from Reynold's averaging. The differences in TKE corresponded to the features found in the brightness temperature. The leftpointing cones substantially enhanced the turbulent exchange at the base of the cones compared to the right-pointing cones (Fig. 5f). Additionally, the right cones strongly reduced TKE in the lee of the cones (Fig. 5f). Both effects caused the left-pointing cones to be subject to enhanced convective heat exchange relative to the right-pointing cones. The right- 

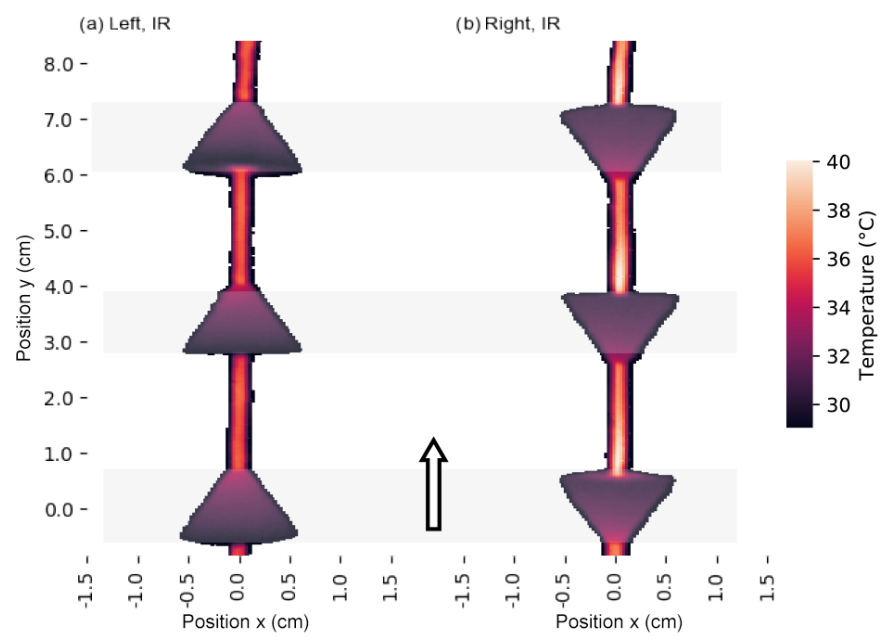

(e) Right

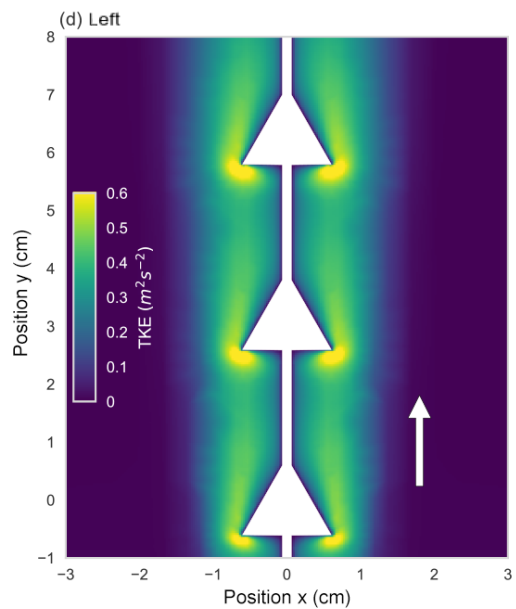

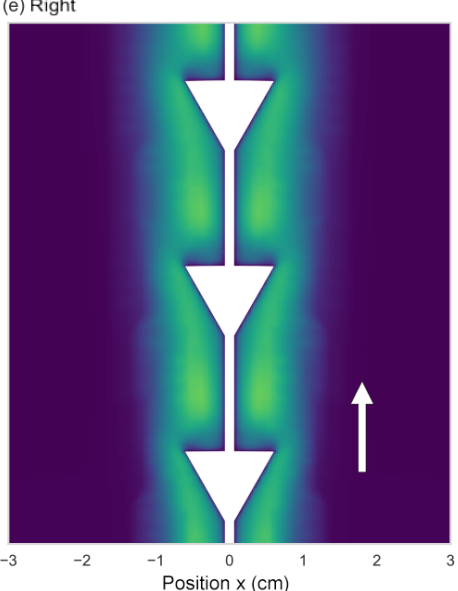
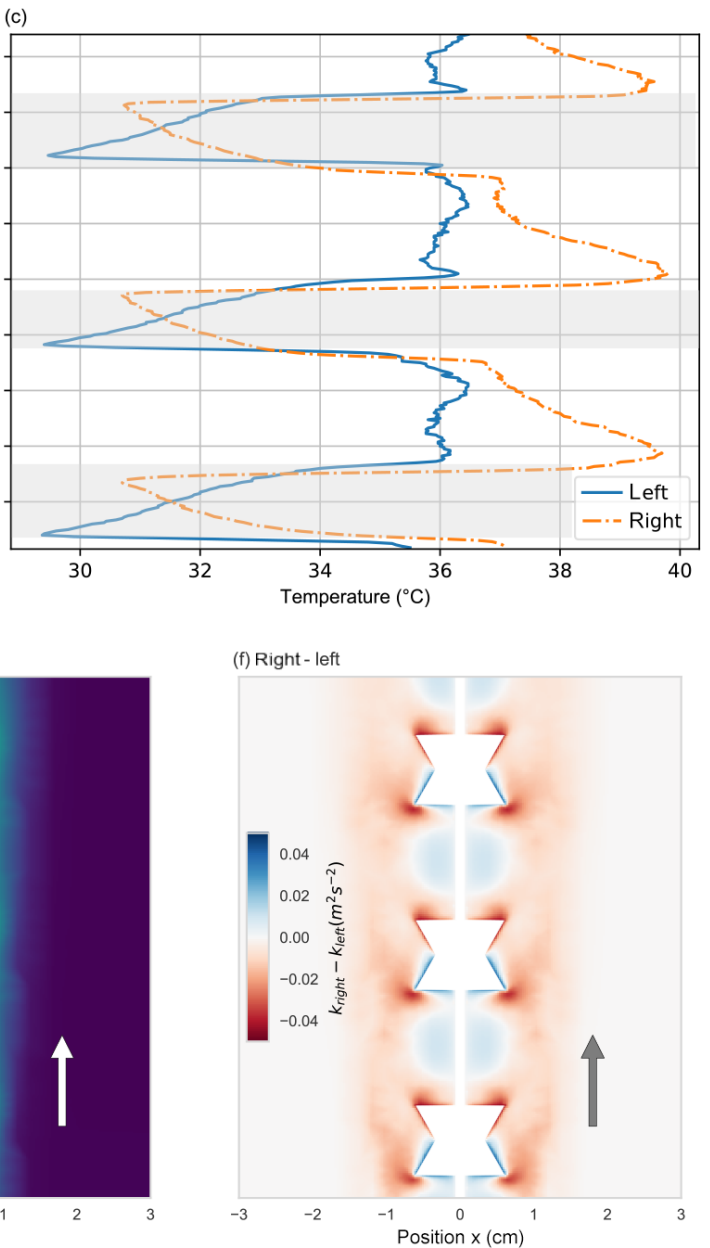

Figure 5. The brightness temperature of the fiber-optic cables with (a) left- and (b) right-pointing cones for the $12 \mathrm{~mm}, 1: 1 \mathrm{cones}$ with $2 \mathrm{~cm}$ spacing and a heating rate of $2.5 \mathrm{~W} \mathrm{~m}^{-1}$ for a wind speed of $0.9 \mathrm{~m} \mathrm{~s}^{-1}$. (c) The average of the three warmest pixels in each horizontal row is provided to better demonstrate the spatial pattern of temperatures along the fiber. The grey shading is to visually line up the cones in (a)-(c). (d, e) Turbulence kinetic energy (TKE) computed from the CFD simulations for the same experiment as in (a) and (b) with (f) the difference in TKE between the two orientations. The difference in TKE was not physically meaningful in the region with a cone in either (d) or (e) and is excluded in (f). The subset arrows indicate the direction of the mean flow.

pointing cones did provide a small enhancement of turbulent exchange further along the fiber, past the region in the lee of the cone (blue colors in Fig. 5f). This enhanced turbulent exchange was also apparent as the brightness temperature of the right-pointing cones decreased from the base of one cone to the tip of the next one (Fig. 5c). However, this small increase in turbulent exchange was not large enough to overcome the reduction in turbulent exchange directly in the lee of the cones. Both the CFD modeling approach and the independent experimental brightness temperatures confirmed that the wind direction signal from the oriented microstructures results from differences in the convective heat exchange generated by the microstructures.

\subsection{Remaining questions and future work}

This study only demonstrated the ability to observe wind direction within one dimension. Convolving the FODS wind direction observations along orthogonal directions into a fully three-dimensional wind field is a substantial challenge and beyond the scope of this proof-of-concept study. The angle of attack of the mean wind direction along the fiber will influence of the wind direction signal, similar to issues with observing wind speed with FODS (Sayde et al., 2015; Pfister et al., 2019; Ramshorst et al., 2019). Exploring the effect of wind attack angle was not possible given the size and aspect ratio of the wind tunnel used in this study. The ability to detect mean wind direction is useful, but developing a flow sensor for studies of atmospheric turbulence also requires the ability to detect rapidly changing wind vectors. 
The cone/cable system has some thermal inertia that creates a lag in reaching an equilibrium $\Delta T$. This study only highlights the ability to measure time- and space-averaged flow, which may not be sufficient to resolve the energy-containing eddies for weak-wind boundary layers. Additional work is necessary for determining the time response of the wind direction signal. Finally, the flow explored in this proof-ofconcept study has a lower turbulence intensity than atmospheric flows. Deployments in real atmospheric flows may require a larger heating rate or further averaging in order to observe a meaningful $\Delta T$. These considerations need to guide future work to enable true three-dimensional observations of wind speed and direction. The method for detecting wind direction depends on the temperature difference between two cables that are identical besides the cone orientation. A number of factors, for instance cable type, size, and the number of optical cores, may cause this temperature difference to vary. Understanding these factors will be critical to developing a robust, empirical relationship between the directionally sensitive temperature signals and wind direction.

\section{Conclusions}

By combining fiber-optic distributed sensing techniques with independent thermal infrared imagery and computational fluid flow simulations, we evaluated and verified a method for detecting distributed wind direction using microstructures affixed to actively heated fiber-optic cables. We demonstrated that the microstructures, which are affixed to a pair of fiber-optic cables in opposing directions, introduce a directional sensitivity of the turbulent heat loss from the cable to the air. This differential convective heat flux can be detected as a temperature difference between the two cables. The temperature difference then allows for computing wind direction along the axis of the fiber, providing a method for observing wind direction on a distributed basis. The work presented here thus represents a critical step in employing the microstructure approach to achieve the ultimate goal of building a spatially resolving, three-dimensional flow sensor for the atmospheric surface layer to record turbulent fluxes of sensible heat and momentum.

Data availability. The data used in this study are available at https://doi.org/10.5281/zenodo.3724596 (Lapo et al., 2020) and include scripts for reproducing the figures in this study.

Author contributions. JS and CT formulated the original concept for this study. All coauthors developed the experimental design. JS built the wind tunnel with further contributions from all coauthors. $\mathrm{KL}, \mathrm{AF}$, and LP performed the analysis and experiments. KL prepared the manuscript with contributions from all coauthors.
Competing interests. The authors declare that they have no conflict of interest.

Acknowledgements. Initial prototyping and testing of the microstructure approach was conducted at Oregon State University, Corvallis, Oregon, at the Openly Published Environmental Sensing Lab (http://www.open-sensing.org, last access: 30 March 2020), with support from Cara Walter, and at the Experimental Fluid Mechanics Research Lab with support from James Liburdy. Chad Higgins provided insights during the original development and testing of the microstructure concept. Further assistance in operating the fiber-optic sensing system in the OSU wind tunnel was provided by Justus van Ramshorst.

Financial support. This project has received funding from the European Research Council (ERC) under the European Union's Horizon 2020 research and innovation programme (grant agreement no. 724629)

This open-access publication was funded by the University of Bayreuth.

Review statement. This paper was edited by Murray Hamilton and reviewed by two anonymous referees.

\section{References}

Cheng, Y., Sayde, C., Li, Q., Basara, J., Selker, J., Tanner, E., and Gentine, P.: Failure of Taylor's hypothesis in the atmospheric surface layer and its correction for eddy- covariance measurements, Geophys. Res. Lett., 44, 4287-4295, https://doi.org/10.1002/2017GL073499, 2017.

Euser, T., Luxemburg, W. M. J., Everson, C. S., Mengistu, M. G., Clulow, A. D., and Bastiaanssen, W. G. M.: A new method to measure Bowen ratios using high-resolution vertical dry and wet bulb temperature profiles, Hydrol. Earth Syst. Sci., 18, 20212032, https://doi.org/10.5194/hess-18-2021-2014, 2014.

Friedrich, K., Lundquist, J. K., Aitken, M., Kalina, E. A., and Marshall, R. F.: Stability and turbulence in the atmospheric boundary layer: A comparison of remote sensing and tower observations, Geophys. Res. Lett., 39, 1-6, https://doi.org/10.1029/2011GL050413, 2012.

Hausner, M. B., Suárez, F., Glander, K. E., and Giesen, N. V. D.: Calibrating Single-Ended Fiber-Optic Raman Spectra Distributed Temperature Sensing Data, Sensors, 11, 10859-10879, https://doi.org/10.3390/s111110859, 2011.

Holtslag, A. A., Svensson, G., Baas, P., Basu, S., Beare, B., Beljaars, A. C., Bosveld, F. C., Cuxart, J., Lindvall, J., Steeneveld, G. J., Tjernström, M., and Van De Wiel, B. J.: Stable atmospheric boundary layers and diurnal cycles: Challenges for weather and climate models, B. Am. Meteorol. Soc., 94, 16911706, https://doi.org/10.1175/BAMS-D-11-00187.1, 2013.

Lapo, K., Freundorfer, A., Pfister, L., Schneider, J., and Thomas, C.: Wind tunnel distributed temperature sensing 
with actively heated fibers and microstructures for detecting wind direction (Version 1.0) [Data set], Zenodo., https://doi.org/10.5281/zenodo.3724596, 2020.

Mahrt, L., Thomas, C. K., and Prueger, J. H.: Space-time structure of mesoscale motions in the stable boundary layer, Q. J. Roy. Meteor. Soc., 135, 67-75, https://doi.org/10.1002/qj.348, 2009.

Owen, P. R. and Thomson, W.: Heat transfer across rough surfaces, J. Fluid Mech., 15, 321-334, https://doi.org/10.1017/S0022112063000288, 1962.

Petrides, A. C., Huff, J., Arik, A., Giesen, N. V. D., Kennedy, A. M., Thomas, C. K., and Selker, J. S.: Shade estimation over streams using distributed temperature sensing, Water Resour. Res., 47, 2-5, https://doi.org/10.1029/2010WR009482, 2011.

Pfister, L., Sigmund, A., Olesch, J., and Thomas, C. K.: Nocturnal Near-Surface Temperature, but not Flow Dynamics, can be Predicted by Microtopography in a MidRange Mountain Valley, Bound.-Lay. Meteorol., 165, 333-348, https://doi.org/10.1007/s10546-017-0281-y, 2016.

Pfister, L., Lapo, K. E., Sayde, C., Selker, J., Mahrt, L., and Thomas, C. K.: Classifying the Nocturnal Atmospheric Boundary Layer into Temperature and Flow Regimes, Q. J. Roy. Meteor. Soc., 145, 1515-1534, https://doi.org/10.1002/qj.3508, 2019.

van Ramshorst, J. G. V., Coenders-Gerrits, M., Schilperoort, B., van de Wiel, B. J. H., Izett, J. G., Selker, J. S., Higgins, C. W., Savenije, H. H. G., and van de Giesen, N. C.: Wind speed measurements using distributed fiber optics: a windtunnel study, Atmos. Meas. Tech. Discuss., https://doi.org/10.5194/amt-2019-63, in review, 2019.

Sayde, C., Thomas, C. K., Wagner, J., and Selker, J.: High-resolution wind speed measurements using actively heated fiber optics, Geophys. Res. Lett., 42, 10064-10073, https://doi.org/10.1002/2015GL066729, 2015.

Schilperoort, B., Coenders-Gerrits, M., Luxemburg, W., Jiménez Rodríguez, C., Cisneros Vaca, C., and Savenije, H.: Technical note: Using distributed temperature sensing for Bowen ratio evaporation measurements, Hydrol. Earth Syst. Sci., 22, 819830, https://doi.org/10.5194/hess-22-819-2018, 2018

Selker, J. S., Thévenaz, L., Huwald, H., Mallet, A., Luxemburg, W., Giesen, N. V. D., Stejskal, M., Zeman, J., Westhoff, M., and Parlange, M. B.: Distributed fiber-optic temperature sensing for hydrologic systems, Water Resour. Res., 42, 1-8, https://doi.org/10.1029/2006WR005326, 2006.

Sigmund, A., Pfister, L., Sayde, C., and Thomas, C. K.: Quantitative analysis of the radiation error for aerial coiled-fiber-optic distributed temperature sensing deployments using reinforcing fabric as support structure, Atmos. Meas. Tech., 10, 2149-2162, https://doi.org/10.5194/amt-10-2149-2017, 2017
Stull, R. B.: An Introduction to Boundary Layer Meteorology, Springer Netherlands, Dordrecht, the Netherlands, 1 edn., 1988.

Sun, J., Mahrt, L., Banta, R. M., and Pichugina, Y. L.: Turbulence Regimes and Turbulence Intermittency in the Stable Boundary Layer during CASES-99, J. Atmos. Sci., 69, 338-351, https://doi.org/10.1175/JAS-D-11-082.1, 2012.

Sun, J., Nappo, C. J., Mahrt, L., Belušic, D., Grisogono, B., Stauffer, D. R., Pulido, M., Staquet, C., Jiang, Q., Pouquet, A., Yagüe, C., Galperin, B., Smith, R. B., Finnigan, J. J., Mayor, S. D., Svensson, G., Grachev, A. A., and Neff, W. D.: Review of wave-turbulence interactions in the stable atmospheric boundary layer, Rev. Geophys., 53, 956-993, https://doi.org/10.1002/2015RG000487, 2015.

Taylor, G. I.: The Spectrum of Turbulence, P. R. Soc. A, 164, 476490, https://doi.org/10.1098/rspa.1938.0032, 1938.

Thomas, C. K.: Variability of Sub-Canopy Flow, Temperature, and Horizontal Advection in Moderately Complex Terrain, Bound.Lay. Meteorol., 139, 61-81, https://doi.org/10.1007/s10546-0109578-9, 2011.

Thomas, C. K., Kennedy, A. M., Selker, J. S., Moretti, A., Schroth, M. H., Smoot, A. R., Tufillaro, N. B., and Zeeman, M. J.: High-resolution fibre-optic temperature sensing: A new tool to study the two-dimensional structure of atmospheric surface layer flow, Bound.-Lay. Meteorol., 142, 177-192, https://doi.org/10.1007/s10546-011-9672-7, 2012.

Tyler, S. W., Selker, J. S., Hausner, M. B., Hatch, C. E., Torgersen, T., Thodal, C. E., and Schladow, S. G.: Environmental temperature sensing using Raman spectra DTS fiber-optic methods, Water Resour. Res., 45, 1-11, https://doi.org/10.1029/2008WR007052, 2009.

Zeeman, M. J., Selker, J. S., and Thomas, C. K.: Near-Surface Motion in the Nocturnal, Stable Boundary Layer Observed with Fibre-Optic Distributed Temperature Sensing, Bound.-Lay. Meteorol., 154, 189-205, https://doi.org/10.1007/s10546-014-99729, 2015.

Zeitoun, O., Ali, M., and Nuhait, A.: International Journal of Thermal Sciences Convective heat transfer around a triangular cylinder in an air cross flow, Int. J. Therm. Sci., 50, 1685-1697, https://doi.org/10.1016/j.ijthermalsci.2011.04.011, 2011. 